\title{
Multiple Order, Multiple-Time Constant Self-Adaptive Tracking Filter
}

\author{
Edison Thomas \\ External Project Consultant, Department of Aerospace, \\ Indian Institute of Science, Bangalore-560012, India
}

\begin{abstract}
The algorithm described provides simultaneous availability of the state estimates corresponding to many orders of filters through the use of the fading memory (discounted) averages of the residuals of each lower order to obtain the estimates of a higher order. These averages are also used to provide maneuver parameters at different levels in order to obtain a gracefully changing hybrid combination of the filter estimates. Further, as the state estimate of a higher order filter is generally better than that of the lower order, particularly in respect of bias errors during and after a maneuver period, continual re-initialization of the lower order filters, using the higher order estimate, is effected through the use of the relevant maneuver parameter, enabling the filter to settle down faster towards the steady state conditions after a maneuver. The self-adaptive use of the the higher order estimates during maneuver thus provides a good smoothing under steady state conditions combined with rapid maneuver following with minimal bias errors, even in low data rate radar systems.
\end{abstract}

\section{INTRODUCTION}

Aircraft targets of most Track While Scan radar systems generally move in long stretches of constant speed trajectories with mild navigational maneuvers, and also with occasional wild maneuvers of short duration. Kalman filters, which optimize the state estimates of these target tracks, against the radar measurement errors and the average maneuver characteristics, perform poorly against severe maneuvers, particularly during maneuver transients. In order to minimize the transient bias errors, that generally occur during and after severe maneuvers, adaptive filters based on short time constants are better suited than those based on variances. The variable dimension filter of Bar Shalom and Birmiwal [1] is an example of the time constant based maneuver detection used for switching among filters of two orders for an improved maneuver following. The filter described in this paper changes gracefully among three filters of different orders with time constant based maneuver detections at two levels, through a simple implementation. The simplicity arises from the use of a novel filter technique, called the Weighted Average Filter Algorithm. In this algorithm, while computing the state estimates of a fourth order filter, the state estimates of a third and second order filter estimates are also available, in addition to the monitoring of the maneuvers, if any, at each measurement update, and this facilitates the adaptive algorithm.

\section{Multiple Order, Multi-time Constant}

As the title of the paper suggests the filter automatically changes, in a self-adaptive manner, among a multiple order of filters based on certain weighted averages with different and varying fading memory time constants. Thus under the conditions of a steady state constant velocity motion of the target the filter essentially operates as a second order (constant velocity) filter with comparatively large time constants for obtaining a good smoothing. When the target trajectory changes suddenly, say for example with a step acceleration, into the dynamics corresponding to a constant acceleration, the filter gradually changes into a third order (constant acceleration) filter with a shorter time constant. During the intermediate period, between the instant of the sudden acceleration and the time at which the filter characteristics approach that of a steady state third order filter, the filter character- istics change into those corresponding to a fourth order filter of even a shorter time constant so as to minimize the maneuver transient errors. When the target motion settles again with a constant velocity characteristics after a maneuver, the filter characteristics change back again gracefully, and automatically into that of a steady state second order filter with large time constants, through intermediate stages of higher order characteristics for minimal transient errors.

\section{Hybrid Combination}

The simultaneous availability of the state vectors of three orders is used for obtaining a hybrid combination of the filter outputs, by providing different relative weights for the different outputs. The filter corrections and the associated time constants change continually and gracefully to change the characteristics of the filter according to the extent of the maneuvers detected.

\section{Maneuver Detection}

The fading memory weighted averages of the residuals, which are used in the estimation of the state parameters of different orders of the filters, also contain sufficient information for providing efficient maneuver detection at different levels for changing the characteristics of the filter among different higher orders as required. Therefore, no extensive additional computations are required for this purpose or for changing back the characteristics to that of lower orders after a maneuver, when the filter tends to settle down towards a steady state constant velocity characteristics.

\section{Self adaption}

The self adaptive nature of the filter arises from the gradual changing of the maneuver detection parameters of the algorithm which gradually and continually change the characteristics of the filter in such a manner as is suited for a good performance against maneuvers and also for the filter to settle down into a constant velocity or constant acceleration characteristics as required. The gradual changes thus provide a graceful change in the filter characteristics, according to the extent of the maneuver detection at different levels.

\section{Gracefully changing Time constants}

The effectiveness of the maneuver detections, to change the characteristics from that of one order into another, through the hybrid weights, is also used for gracefully changing the time constants of the filters in such a manner as to effect a comparatively quicker recovery towards the steady state conditions during and after a maneuver. This provides an additional dimension to the adaptivity of the filter characteristics towards the desirable performance.

\section{Variance reduction}

As the time constants that are operational, under different conditions of the target dynamics, are mainly chosen for a good maneuver following characteristics, the variance reduction ratios obtainable for the state parameters also vary and are related to the number of updates of the measurement data within the relevant time constants. 
Thus while comparing the filter performance, through simulation, of the results of two filters with the same time constants, but with different measurement data update rates, it may be readily seen that though the maneuver following performance is nearly the same in both these cases, the smoothing is better for the case of the filter operating on the higher data update rate. Therefore, while the time constants of the filter algorithm are chosen in accordance with the requirements of a good maneuver following, the filter output accuracy can be improved only through a better measurement accuracy for the system and/or a higher data-rate as required. Computation of the expected variance reduction ratio for the various parameters under the steady state conditions for the various orders of the filters can be readily carried out analytically. However, the procedure for the calculations is generally algebraically intensive. An alternate method of obtaining the variance reduction ratios is through Monte Carlo analysis, under simulated conditions.

\section{Variable time updates}

Modern radars, particularly those using phased arrays, generally have different target update rates for different targets, and even for the same target during the different stages of its track life. The algorithm readily provides for such changes in the time updates, and yet maintain the characteristics of the time constants designed for the filter algorithm. This provision can also be used with the constant scan rate radars, for handling plot updates after missed detections in certain scans of the radar. It can also be used for changes in the time between hits, due to rapid relative target motion in azimuth, provided the clock time of the hits are available along with the plot data. The advantage of being able to handle any time duration between hits is particularly very useful for airborne radars for handling changes in scan intervals during platform turns.

The algorithm of the fading memory weighted average residual filter is described in the following section. Appendices support the description with relevant mathematical details. The implementation of the algorithm as a self adaptive filter in a single dimension is described in the next section followed by the simulation results on a typical target trajectory. Practical applications of the algorithm for multi-dimensional cases is described in the last section.

\section{WEIGHTED AVERAGE FILTER ALGORITHM}

\section{Fading Memory Weighted averages}

If $V_{n}$ is a quantity available at the $n^{\text {th }}$ instant of a semi-infinite sampled time series, its fading memory weighted average $W_{n}$, with respect to the fading memory parameter $\lambda_{1}$ is defined as

$$
\begin{aligned}
W_{n} & =\frac{\sum_{k=0}^{\infty} V_{n-k} \lambda_{1}^{k}}{\sum_{k=0}^{\infty} \lambda_{1}^{k}} \\
& =\left(1-\lambda_{1}\right) \sum_{k=0}^{\infty} V_{n-k} \lambda_{1}^{k}
\end{aligned}
$$

The value $V_{n-k}$ corresponds to the instant $k T$ earlier to the current $\left(n^{t h}\right)$ instant.

The weighted average may be recursively updated using the previous weighted average and the current value of $V_{n}$

$$
W_{n}=W_{n-1} \lambda_{1}+V_{n}\left(1-\lambda_{1}\right)
$$

Such a weighted average will be generally denoted hereafter as

$$
W_{n}=\overline{V_{n}}\left(\lambda_{1}\right)
$$

\section{Time constant}

If the time interval between the successive samples of the series is $T$, the time constant $T_{1}$, associated with $W_{n}$ is given by the time average of the samples. Therefore

$$
T_{1}=\frac{\sum_{k=0}^{\infty} k T \lambda_{1}^{k}}{\sum_{k=0}^{\infty} \lambda_{1}^{k}}=\frac{\lambda_{1} T}{1-\lambda_{1}}
$$

The inverse relation gives the fading memory constant $\lambda_{1}$ to be associated with a given time constant $T_{1}$, as

$$
\lambda_{1}=\frac{T_{1}}{T_{1}+T}=\frac{t_{1}}{t_{1}+1}
$$

where $t_{1}=T_{1} / T=\lambda_{1} /\left(1-\lambda_{1}\right)$ is defined as the normalized time constant.

\section{The Weighted Average Filter}

The theory of Weighted Average Filter is developed in Appendix A, but the gist of the algorithm is presented here, with respect to a single dimension in space, for illustrating the performance. The state vector components at the $n^{\text {th }}$ instant are assumed in terms of the position component and its backward differences, [2] instead of the more conventional form in terms of the position component and its time derivatives.

$$
X_{n}=\left[\begin{array}{llll}
x & \nabla x & \nabla^{2} x & \nabla^{3} x
\end{array}\right]_{n}^{T}
$$

The state vector thus defined in terms of the finite differences is better suited for mathema,tical analysis. However, the velocity and acceleration components, if required, may be readily obtained by the methods of finite differences as

$$
\left[\begin{array}{c}
x \\
\dot{x} \\
\ddot{x} \\
\ddot{x}
\end{array}\right]_{n}=\left[\begin{array}{cccc}
1 & 0 & 0 & 0 \\
0 & \frac{1}{T} & \frac{1}{2 T} & \frac{1}{3 T} \\
0 & 0 & \frac{1}{T^{2}} & \frac{1}{2 T^{2}} \\
0 & 0 & 0 & \frac{1}{T^{3}}
\end{array}\right]\left[\begin{array}{c}
x \\
\nabla x \\
\nabla^{2} x \\
\nabla^{3} x
\end{array}\right]_{n}
$$

\section{First order filter}

The first weighted average estimate $\hat{A}_{n}$ is the weighted average of the position measurements $x_{M}$

$$
\hat{A}_{n}=\overline{x_{M n}}\left(\lambda_{1}\right)
$$

$\hat{A}_{n}$ represents the first order filter position estimate, $\hat{x}_{1_{n}}$ for a static target, which is smoothed through the fading memory averaging of the current and the past measurements.

\section{Second order filter}

However, for a target moving at a constant speed, this average would lag behind the current position by a constant lag. A smoothed estimate of this lag is obtained by the weighted average of the first order filter residual defined by

$$
\begin{aligned}
\hat{L}_{n} & =\overline{x_{M n}-\hat{x}_{1 n}}\left(\lambda_{2}\right) \\
& =\overline{x_{M n}-\hat{A}_{n}}\left(\lambda_{2}\right)
\end{aligned}
$$

A different fading memory parameter $\lambda_{2}$, corresponding to a different time constant $T_{2}$, is used for generality. The second order filter estimates $\hat{x}_{2}$ and $\hat{V} x_{2}$ corresponding to position and first difference components are obtainable from the two weighted averages $\hat{A}_{n}$ and $\hat{L}_{n}$ as

$$
\left[\begin{array}{c}
\hat{x}_{2} \\
\hat{\nabla} x_{2}
\end{array}\right]_{n}=\left[\begin{array}{cc}
1 & 1 \\
0 & \frac{1}{t_{1}}
\end{array}\right]\left[\begin{array}{c}
\hat{A}_{n} \\
\hat{L}_{n_{2}}
\end{array}\right]
$$

\section{Third order filter}

The second order position estimate would tend to have a constant dynamic lag, at the steady state, for a target moving with a constant acceleration. A smonthed estimate of this dynamic lag may be obtained by a third weighted average, of the second order filter residuals :-

$$
\begin{aligned}
\hat{L}_{n} & =\overline{\left(x_{M n}-\hat{x}_{2 n}\right)}\left(\lambda_{3}\right) \\
& =\frac{\left(x_{M n}-\left(\hat{A}_{n}+\hat{L}_{n}\right)\right)}{\left(\lambda_{3}\right)}
\end{aligned}
$$

Here again a different parameter $\lambda_{3}$ is used for generality. The third order filter estimates for position, first difference and second difference 
components are obtainable from

$$
\left[\begin{array}{c}
\hat{x}_{3} \\
\hat{\nabla} x_{3} \\
\hat{\nabla}^{2} x_{3}
\end{array}\right]_{n}=\left[\begin{array}{ccc}
1 & 1 & 1 \\
0 & \frac{1}{t_{1}} & \frac{1}{t_{1}}+\frac{1}{t_{2}} \\
0 & 0 & \frac{1}{t_{1} t_{2}}
\end{array}\right]\left[\begin{array}{c}
\hat{A}_{n} \\
\hat{L}_{n} \\
\hat{D}_{n}
\end{array}\right]
$$

Even if the acceleration lasts only for a short duration, the third order filter performance is better than that of the second order filter from the point of view of maneuver following. However, the third order filter has a comparatively poorer smoothing, and therefore its use should be limited to a short period during and after a maneuver acceleration, for optimising the filter performance.

\section{Fourth order filter}

Extending the principle further, a fourth order filter estimate may be similarly obtained through the weighted average estimate of the bias error of the third order filter output, through the weighted average of the third order residuals

$$
\begin{aligned}
\hat{E}_{n} & =\overline{\left(x_{M n}-\hat{x}_{3 n}\right)}\left(\lambda_{4}\right) \\
& =\left(x_{M n}-\left(\hat{A}_{n}+\hat{L}_{n}+\hat{D}_{n}\right)\right)\left(\lambda_{4}\right)
\end{aligned}
$$

The fourth order filter estimates are given by

$$
\left[\begin{array}{c}
\hat{x}_{4} \\
\hat{\nabla} x_{4} \\
\hat{\nabla}^{2} x_{4} \\
\hat{\nabla}^{3} x_{4}
\end{array}\right]_{n}=\left[\begin{array}{cccc}
1 & 1 & 1 & 1 \\
0 & \frac{1}{t_{1}} & \frac{1}{t_{1}}+\frac{1}{t_{2}} & \frac{1}{t_{1}}+\frac{1}{t_{2}}+\frac{1}{t_{3}} \\
0 & 0 & \frac{1}{t_{1} t_{2}} & \frac{1}{t_{1} t_{2}}+\frac{1}{t_{2} t_{3}}+\frac{1}{t_{3} t_{1}} \\
0 & 0 & 0 & \frac{1}{t_{1} t_{2} t_{3}}
\end{array}\right]\left[\begin{array}{c}
\hat{A}_{n} \\
\hat{L}_{n} \\
\hat{D}_{n} \\
\hat{E}_{n}
\end{array}\right]
$$

The fourth order filter generally provides superior maneuver following capability over the third order filter during and after a severe maneuver but the smoothing is comparatively poorer as is to be expected, and therefore has to be used only for a short duration, during the maneuver transients.

\section{Hybrid combination}

Equations (10), (12) and (14) may be combined into a single equation in the form :-

$$
\left[\begin{array}{c}
\hat{x}_{h} \\
\hat{\nabla} x_{h} \\
\hat{\nabla}^{2} x_{h} \\
\hat{\nabla}^{3} x_{h}
\end{array}\right]_{n}=\left[\begin{array}{cccc}
1 & 1 & 1 & 1 \\
0 & \frac{1}{t_{1}} & \frac{1}{t_{1}}+\frac{1}{t_{2}} & \frac{1}{t_{1}}+\frac{1}{t_{2}}+\frac{1}{t_{3}} \\
0 & 0 & \frac{1}{t_{1} t_{2}} & \frac{1}{t_{1} t_{2}}+\frac{1}{t_{2} t_{3}}+\frac{1}{t_{3} t_{1}} \\
0 & 0 & 0 & \frac{1}{t_{1} t_{2} t_{3}}
\end{array}\right]\left[\begin{array}{c}
\hat{A}_{n} \\
\hat{L}_{n} \\
h_{1} \hat{D}_{n} \\
h_{2} \hat{E}_{n}
\end{array}\right]
$$

where, the constants $h_{1}, h_{2}$ are termed the hybrid parameters and the state vector estimate is defined as the hybrid filter estimate.

It can be readily shown that

$$
\left[\begin{array}{c}
\hat{x}_{h} \\
\hat{\nabla} x_{h} \\
\hat{\nabla}^{2} x_{h} \\
\hat{\nabla}^{3} x_{h}
\end{array}\right]_{n}=\left[\begin{array}{c}
\hat{x}_{2} \\
\hat{\nabla} x_{2} \\
0 \\
0
\end{array}\right]_{n}\left(1-h_{1}\right)+\left[\begin{array}{c}
\hat{x}_{3} \\
\hat{\nabla} x_{3} \\
\hat{\nabla}^{2} x_{3} \\
0
\end{array}\right]_{n}\left(h_{1}-h_{2}\right)+\left[\begin{array}{c}
\hat{x}_{4} \\
\hat{\nabla} x_{h} \\
\hat{\nabla}^{2} x_{h} \\
\hat{\nabla}^{3} x_{h}
\end{array}\right]_{(16)} h_{2}
$$

Therefore, the name hybrid filter arises from the fact that the estimate is nothing but a weighted average of the second order, third order and fourth order filters described earlier, with relative weights $\left(l-h_{1}\right)$, $\left(h_{1}-h_{2}\right)$ and $\left(h_{2}\right)$. When $h_{1} \rightarrow 0$ and $h_{2} \rightarrow 0$, the hybrid output tends towards the second order filter output. When $h_{1} \rightarrow 1$ and $h_{2} \rightarrow 0$, the hybrid output tends towards the third order filter output. Then $h_{1} \rightarrow 1$ and $h_{2} \rightarrow 1$ the hybrid filter tends towards the fourth order filter output. Thus by controlling the hybrid parameters, the hybrid filter characteristics can be changed from that of a second order filter, to a third order filter or to a fourth order filter through graceful intermediate stages. This principle may be used for the adaptive changing of the filter characteristics during and after a maneuver.

\section{Maneuver Detection}

Assume that the measurement errors on the position $x_{M}$ are Gaussian with a constant variance. When the target moves with constant velocity, under the steady state conditions, the residuals of the second order filter have a zero mean constant variance characteristics. Therefore, the weighted average of the second order residuals $\hat{D}_{n}$, also, has a zero mean constant variance characteristics. The value of the constant variance, as worked out in the Appendix, is given by

$$
\sigma_{D}^{2}=K_{D} \sigma_{x}^{2}
$$

where $K_{D}$ is the variance reduction factor associated with $D_{n}$ given by

$$
K_{D}=\frac{\left(1-\lambda_{3}\right)^{2} \lambda_{1}^{2} \lambda_{2}^{2}\left[8\left(1+\lambda_{1} \lambda_{2} \lambda_{3}\right)-2\left(1+\lambda_{1}\right)\left(1+\lambda_{2}\right)\left(1+\lambda_{3}\right)\right]}{\left(1+\lambda_{1}\right)\left(1+\lambda_{2}\right)\left(1+\lambda_{3}\right)\left(1-\lambda_{1} \lambda_{2}\right)\left(1-\lambda_{2} \lambda_{3}\right)\left(1-\lambda_{3} \lambda_{1}\right)}
$$

However, when the target begins to maneuver, say with a step acceleration, $\hat{D}_{n}$ no longer has zero mean characteristics as it develops a bias which tends to build up towards the steady state dynamic lag value corresponding to the acceleration. Thus the statistically significant non-zero value of $\hat{D}_{n}$ is by itself an indication of the maneuver which can be used for developing a suitable acceleration detection. The value $h_{1}$ of the hybrid parameter can be changed from a near zero value under static condition to a near unity value during the period of an acceleration, based on such an acceleration detector. An empirical function for $h_{1}$, to meet the above requirements is

$$
h_{1}=\frac{\hat{D}_{n}^{2}}{\hat{D}_{n}^{2}+k_{1} \sigma_{D}^{2}}
$$

A typical value for $k_{1}$, is $25 . \quad k_{1} \sigma_{D}^{2}$ can be considered as a threshold value for $\hat{D}_{n i}^{2}$. When $D_{n}^{2}$ becomes equal to this threshold, $h_{1}$ has value 0.5 . When it crosses the threshold, $h_{1}$ rapidly tends towards unity, when it is smaller than the threshold $h_{1}$, tends towards zero. Thus the use of the hybrid parameter $h_{1}$, based on the value of $D_{n}$ serves as an excellent acceleration detector to change the second order filter characteristics to that of the third order.

Similarly the value of $E_{n}$ has zero mean, constant variance characteristics when the target is in steady state constant velocity or constant acceleration conditions. The variance value may be written in terms of the variance reduction ratio $K_{E}$ associated with $E_{n}$ as

$$
\sigma_{E}^{2}=K_{E} \sigma_{x}^{2}
$$

The value of $K_{E}$, as obtained through extensive algebraic calaculation, is given in the Appendix as it is too large to be given here. However once it is pre-computed, it can be used for obtaining a higher level maneuver detection through the second hybrid constant $h_{2}$ is obtained in a similar manner as $h_{1}$.

$$
h_{2}=\frac{\hat{E}_{n}^{2}}{\hat{E}_{n}^{2}+k_{2} \sigma_{E}^{2}}
$$

Typical value for $k_{2}$ is also 25 . Thus the weighted averages $D_{n}$ and $E_{n}$ used for obtaining the higher order state parameters are also used for obtaining the self adaptive hybrid parameters which tend towards unity during and after a maneuver and tend to become zero when the filter tends to change back into the lower order steady state conditions.

\section{Choice of Time Constants}

The parameters $\lambda_{1}, \lambda_{2}, \lambda_{3}, \lambda_{4}$ assumed for the weighted averages are obtained, using equation (5), based on specially chosen time constants $T_{1}, T_{2}, T_{3}, T_{4}$ for a good filter performance. $T_{1}$ and $T_{2}$ are chosen sufficiently large, in order to obtain good smoothing characteristics, and yet small in comparison with the average period of constant velocity motion of the target, between maneuvers, so that the filter settles down into steady state constant velocity characteristics over most parts of the constant velocity segments of the target. $T_{3}$ on the other hand while required to be large for a good smoothing, is required to be small in comparison with the average periods of mild maneuvers, 
in the order of minutes, so that the dynamic lag, which builds up is quickly compensated so that the filter tends to reach the steady state constant acceleration condition over most parts of the constant acceleration segments. $T_{4}$ is selected as a comparatively shorter time constant for quickly compensating for the residual bias errors. No attempt is made to maintain the variance reduction ratios of the filter estimates small during the operation of the filter, when under the fourth order characteristics, as the object is to quickly minimize the bias errors which can become very large in comparison with the measurement errors during severe maneuvers. Thus the time constant based maneuver following characteristics are mainly aimed at rapid maneuver following capabilities, independent of the filter variances and covariances.

\section{Interpretation of $E_{n}$}

A fourth order filter is basically suited for a target dynamics with a constant rate of change of acceleration. However, in a typical scenario of a target moving in segments of constant velocity and constant acceleration, the value of $E_{n}$ represents an estimate of the bias error of a third order filter during maneuvers. The addition of terms involving $E_{n}$ in the estimate of $\hat{x_{n}}, \hat{\nabla} x_{h}$ and $\hat{\nabla}^{2} x_{h}$ in equation (15) thus represents a reduction in the bias errors in these terms with respect to a third order filter, which results in improved estimate of the state vector components during and after a maneuver, particularly when the time constant associated with $E_{n}$ is small.

\section{Rapid Maneuver Correction}

Although the hybrid filter estimates obtained from (15) after updating the weighted averages $A, L, D$ and $E$ from $(8,9,11$ and 13$)$ provide a good state estimate, during and after a maneuver, the filter basically operates with poor smoothing, during the period when $h_{2}$ is significant, lasting upto several time constants of the lower order filters, in which these gradually settle down towards their steady states. This causes comparatively poor smoothing characteristics over this long period, particularly as the lower order filters are generally selected with large time constants for a good smoothing. However, noting that the terms containing $E_{n}$ in (15) contribute to a better estimate of the state vector with lesser bias errors, the third order hybrid filter estimates can be augmented by these terms and the filter correspondingly re-initialised for rapid maneuver correction. The correction required in $\hat{x}, \hat{\nabla} x$ and $\hat{\nabla}^{2} x$ terms for this is given by:

$$
\left[\begin{array}{c}
\delta \hat{x_{3}} \\
\delta \hat{\nabla} x_{3} \\
\delta \hat{\nabla}^{2} x_{3}
\end{array}\right]_{n}=\left[\begin{array}{c}
1 \\
\frac{1}{t_{1}}+\frac{1}{t_{2}}+\frac{1}{t_{3}} \\
\frac{1}{t_{1} t_{2}}+\frac{1}{t_{2} t_{3}}+\frac{1}{t_{3} t_{1}}
\end{array}\right]_{n} h_{2} E_{n}
$$

As the corrections upto $h_{2} E$ are already incorporated thus on the third order filter, the balance $\left(1-h_{2}\right) E$ is left in place of $E$ for further update and correction in the subsequent filter updates. The improvement in the filter performance is even more effective when the time constants $t_{1}, t_{2}, t_{3}$ in eqn (22) are decreased during maneuver by a maneuver dependent factor $k h_{2}$ where $k$ is typically given a value of 2 or 3 . This factor enhances the corrections when $k h_{2}>1$ (during maneuver) and reduces its effect when $k h_{2}<1$ (when the filter approaches steady state conditions). Thus the filter adapts itself more rapidly during maneuver while the variance reduction under steady state is not significantly affected. The corrections required in the state estimate thus becomes

$$
\left[\begin{array}{c}
\delta \hat{x_{3}} \\
\delta \hat{\nabla} x_{3} \\
\delta \hat{\nabla}^{2} x_{3}
\end{array}\right]_{n}=\left[\begin{array}{c}
1 \\
k h_{2}\left(\frac{1}{t_{1}}+\frac{1}{t_{2}}+\frac{1}{t_{3}}\right) \\
k^{2} h_{2}^{2}\left(\frac{1}{t_{1} t_{2}}+\frac{1}{t_{2} t_{3}}+\frac{1}{t_{3} t_{1}}\right)
\end{array}\right]_{n} h_{2} E_{n}
$$

The corresponding changes in $A L D$ are given by

$$
\left[\begin{array}{l}
\delta A \\
\delta L \\
\delta D
\end{array}\right]_{n}=\left[\begin{array}{ccc}
1 & -t_{1} & t_{1}^{2} \\
0 & t_{1} & -t_{1}\left(t_{1}+t_{2}\right) \\
0 & 0 & t_{1} t_{2}
\end{array}\right]\left[\begin{array}{c}
1 \\
k h_{2}\left(\frac{1}{t_{1}}+\frac{1}{t_{2}}+\frac{1}{t_{3}}\right) \\
k^{2} h_{2}^{2}\left(\frac{1}{t_{1} t_{2}}+\frac{1}{t_{2} t_{3}}+\frac{1}{t_{3} t_{1}}\right)
\end{array}\right]_{n} h_{2} E_{n}
$$

Refer equation (A-17) in the Appendix. After re-initialising $A, L$ and $D$ the state parameters are obtained using (12).

\section{Second Order Re-initialisation}

For low data rate surveillance radars only the position and velocity estimates are required. For severe maneuvers of short duration, $D_{n}$ may be treated as a bias error of the second order filter with respect to the 'step velocity' like target characteristics. An improved second order characteristics may be obtained by re-initialising $A$ and $L$ with the above third order estimates. The corrections required are

$$
\left[\begin{array}{c}
\delta \hat{x}_{2} \\
\delta \hat{\nabla} x_{2}
\end{array}\right]_{n}=\left[\begin{array}{c}
1 \\
h_{1} / t_{1}
\end{array}\right]_{n} h_{1} \hat{D}_{n}
$$

The corresponding further changes in $A$ and $L$ are obtained using the matrix $\left[\begin{array}{cc}1 & -t_{1} \\ 0 & t\end{array}\right]$, and the second order estimates using (10). $D_{n}$ is replaced by the uncorrected part $\left(1-h_{1}\right) D_{n}$.

\section{Predicted Position}

Predicted parameters are not used in the filter algorithm. However, the one step predictied position may be obtained, for data association at the next scan, using

$$
\begin{aligned}
x_{p} & =x+\hat{\nabla} x+\hat{\nabla}^{2} x \\
\text { or } x_{p} & =x+\hat{\nabla} x
\end{aligned}
$$

depending on whether the re-initialisation is upto the third order or second order respectively.

\section{IMPLEMENTATION OF THE FILTER}

The efficacy of the filter is now illustrated through the implementation of a filter and its performance evaluation on a simulated target. The filter is designed based on the following parameters:

$$
\begin{aligned}
& T 1=60 \mathrm{sec} ; T 2:=60 \mathrm{sec} ; T 3=10 \mathrm{sec} ; T 4=5 \mathrm{sec} ; \\
& k 1=k 2=25 ; \sigma_{s:}=100 \mathrm{~m} ; k=3
\end{aligned}
$$

A target at init:ial position $150 \mathrm{Km}$ is simulated to move with an initial speed of $300 \mathrm{~m} / \mathrm{sec}$ for a period of 200 seconds. The target is then made to unde:go a linear accelerations of magnitude $10 \mathrm{~m} / \mathrm{sec}^{2}$ for a duration 40 seconds. The target is assumed to continue at the constant speed picked up by the acceleration after the maneuver. The target position corresponding to each update after an interval ' $T$ ' is determined and is provided, along with an added measurement error of variance $\sigma_{x}^{2}$ in order to obtain the measurement sequence. The weight parameters corresponding to the time constants and the update interval $T$ and the corresponding values of $K_{D}$ and $K_{E}$ are precomputed. The results are given for $T=10$ secs and $T=2$ secs for comparison.

\section{The Basic Algorithm}

$A$ and $L$ are initialized based on the least squares fit for the position and the first difference components on the first three measurement data. $D$ and $E$ are initialized with zero values. The filter equations are executed sequentially after each new measurement update, using the previous weighted averages in a recursive manner, and therefore the indices $n$, and $n-1$ are omitted, with the assumption that the computations are carried out in the sequence given and that the latest computed values are available in each computational step.

$$
\begin{aligned}
A= & A \lambda_{1}+x_{M}\left(1-\lambda_{1}\right) \\
L= & L \lambda_{2}+\left(x_{M}-A\right)\left(1-\lambda_{2}\right) \\
D= & \left.D \lambda_{3}+\left(x_{M}-A-L\right)\right)\left(1-\lambda_{3}\right) \\
\boldsymbol{E}= & \left.E \lambda_{4}+\left(x_{M}-A-L-D\right)\right)\left(1-\lambda_{4}\right) \\
& h_{2}=\frac{\left(E^{2}\right)}{\left[\left(E^{2}\right)+\left(k_{2} K_{E} \sigma_{x}^{2}\right)\right]}
\end{aligned}
$$


Re-initialisation of $A L$ and $D$ - The changes in $A L$ and $D$ as required are obtained using equation (24) as the weighted averages and re-initialised as follows.

$$
\begin{aligned}
E & =\left(1-h_{2}\right) E \\
D & =D+\delta D \\
L & =L+\delta L \\
A & =A+\delta A \\
h_{1} & =\frac{\left(D^{2}\right)}{\left[\left(D^{2}\right)+\left(k_{1} K_{D} \sigma_{x}^{2}\right)\right]} \\
x_{3 h}= & A+L+h_{1} D \\
\nabla x_{3 h}= & L / t_{1}+h_{1} D / t_{1}+h_{1} D / t_{2} \\
\nabla^{2} x_{3 h}= & h_{1} D / t_{1} t_{2}
\end{aligned}
$$

Second Order Re-initialisation - As the results are to demonstrate the performance of a low data rate filter, further changes required on $A$ and $L$ for second orderr re-initialisation are carried out and the improved second order estimates $x_{E}$ and $\hat{\nabla} x_{E}$ are obtained as described earlier, and converted to $\hat{x}_{E}$ and $\dot{\hat{x}}_{E}$ parameters.

\section{RESULTS}

The performance evaluation of the filter for the two data rates specified, are given in the form of Monte Carlo averages of 1000 independent runs, each with a different error sequence, in Fig. 1. The mean values of the hybrid parameters $h_{1}$ and $h_{2}$ are also given in the time scale to show the self-adaptive nature of the filter, during and after the step maneuver acceleration. It may be seen that the maneuver following characteristics are nearly the same for the two cases but the variance reduction is better in the filter with the faster update.

\section{FIG .1. FILTER PERFORMANCE}

Target acceleration $10 \mathrm{~m} / \mathrm{sec}^{2}$ at $200 \mathrm{sec}$ for $40 \mathrm{sec}$.

$$
\begin{aligned}
& \text { Update time } T=10 \text { seconds } \\
& \text { Update time } T=2 \text { seconds }
\end{aligned}
$$

Filter reinitialised upto $2^{\text {nd }}$ order.

H2 : Second hybrid parameter

H1 : First hybrid parameter

$$
\begin{aligned}
\text { MDRX : Normalised Mean Deviation (Position) } & \\
& =\frac{\text { Mean Deviation in } \hat{x} \text { w.r.t. true } x}{\sigma_{x}}
\end{aligned}
$$

VRRX : Variance Reduction Ratio (Position) $=($ Variance of $\hat{x}) / \sigma_{x}^{2}$

MDRXD : Normalised Mean Deviation (Velocity) $=$ Mean Deviation in $\hat{\hat{x}}$ w.r.t. true $\dot{x}$

$$
\left(\sigma_{x} / T\right)
$$

VRRXD: Variance Redyction Ratio (Velocity) $=($ Variance of $\hat{\mathrm{x}}) /\left(\sigma_{x} / T\right)^{2}$

For target acceleration $40 \mathrm{~m} / \mathrm{sec}^{2}$ for 10 seconds performance is similar except that the peak values of MDRX, MDRXD reach -6.3 and -26.9 ; respectively for $T=10$ seconds, at one scan after the acceleration.
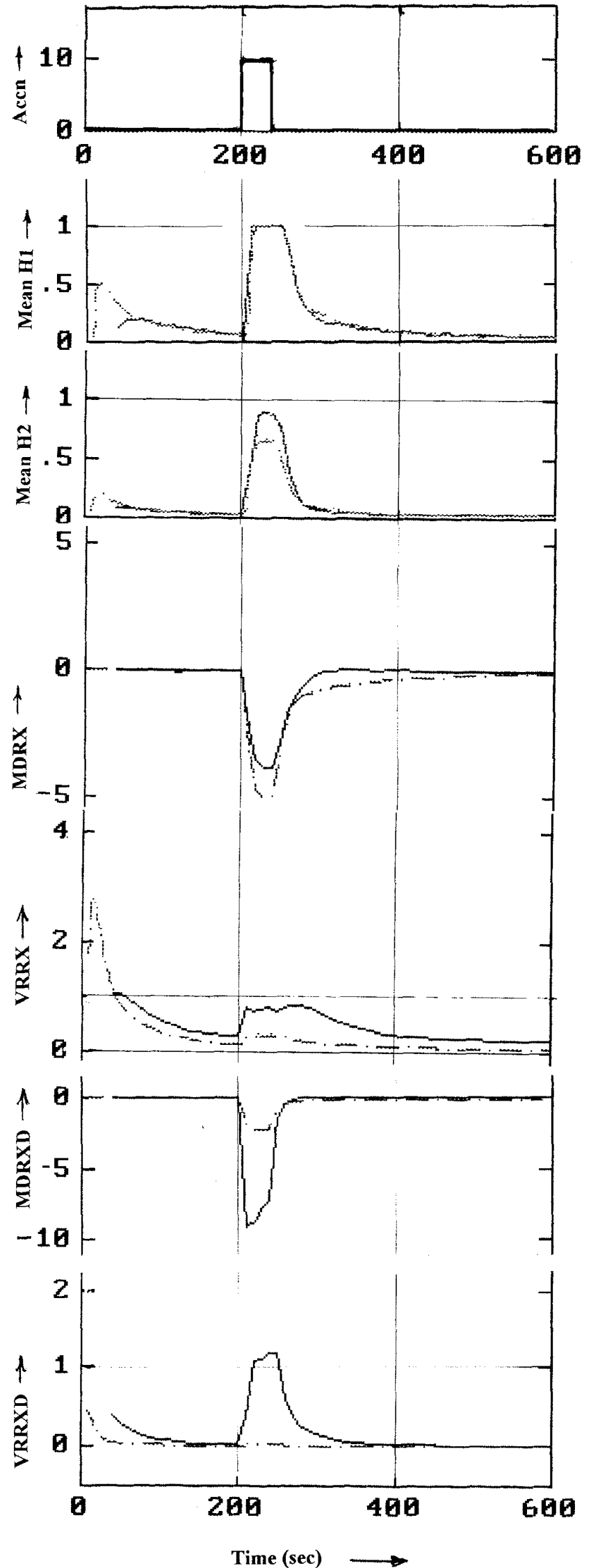
PRACTICAL APPLICATIONS

FOR A MULTI-DIMENSIONAL FILTERING

When operating under two or more dimensions, the state vector components are treated as independent in the two directions and the weighted averages are generated and updated for each dimension separately. However the filter time constants and the hybrid parameters used are the same for all the dimensions. The hybrid parameters are determined based on a joint maneuver detector based on a $\chi^{2}$ threshold on the number of dimensions used.

For example, if a two dimensional filter in Cartesian coordinates is used, the corresponding joint maneuver parameters $h_{1}$ and $h_{2}$ are

$$
\begin{aligned}
& h_{1}=\frac{\left(\frac{D_{x}^{2}}{\sigma_{x}^{2}}+\frac{D_{y}^{2}}{\sigma_{y}^{2}}\right)}{\left(\frac{D_{x}^{2}}{\sigma_{x}^{2}}+\frac{D_{y}^{2}}{\sigma_{y}^{2}}\right)+K_{D} \chi^{2}(2)} \\
& h_{2}=\frac{\left(\frac{E_{x}^{2}}{\sigma_{x}^{2}}+\frac{E_{y}^{2}}{\sigma_{y}^{2}}\right)}{\left(\frac{E_{x}^{2}}{\sigma_{x}^{2}}+\frac{E_{y}^{2}}{\sigma_{y}^{2}}\right)+K_{E} \chi^{2}(2)}
\end{aligned}
$$

The typical $\chi^{2}(2)$ values in the order of 25 provide a good threshold performance.

The use of the common hybrid parameters and the same time constants in all the dimensions produce the same over all variance reduction ratios, at each measurement update, in all the dimensions. Thus the shape of the error ellipsoid of the smoothed estimates is proportional to that of the measurement error ellipsiod, as the effect on the variances and covariances, due to the correlation between the measurement coordinates, is also carried into the weighted averages.

The joint maneuver detection based on the $\chi^{2}$ threshold enables earlier detection and correction, as the maneuvers are generally correlated in spatial dimensions. This is particularly more so when the measurement variances are nearly equal in the two dimensions.

When the variances differ widely, the maneuver detection in the dimension of smaller variance enables a quicker correction also in the dimension with poorer accuracy.

Variable Time Update

When the time interval $T$ is to be changed, the corresponding weight parameters $\lambda_{1}, \lambda_{2}, \lambda_{3}, \lambda_{4}$ are re-computed. $K_{D}, K_{E}$ are also recomputed if the change in $T$ is more than marginal. The weighted averages $A, L, D, E$ are then re-initiated, with the current state vector and the new normalized time-constants, using equation (A17), before using the filter equations.

\section{CONCLUSION}

Weighted average filters based on multiple time constants provide simultaneous availability of the outputs of different orders along with maneuver detector parameters which provide simple self adaptive algorithms with good maneuver following capabilities. The time constants specially chosen for maneuver following are independent of the data rate and the measurement accuracies. The variance reduction ratios which may be precomputed for the steady state conditions of the target, as constants, however vary during different stages of maneuver but the bias errors during the maneuver transients and the subsequent time for approaching steady state conditions are reduced rapidly with the adaptivity of the filter. This last effect is specially advantageous for weapon control radar systems as the target state vector during the maneuver, is mostly close to the true state vector.

\section{ACKNOWLEDGEMENT}

The author is thankful to the Defence Research and Development Organisation (DRDO), and the Indian Institute of Science (IISc) for the encouragement and support given. He is, in particular, grateful to Dr. K. Ramchand, Director, Centre for Airborne Systems, Bangalore and Prof. N. Balakrishnan, Chairman, SERC, IISc. He also wishes to acknowledge his son, Dr. Peter Thomas of Brookehaven National Laboratory, Long Island, for the confirmation of the long derivations.

\section{REFERENCES}

[1] BarShalom and K. Birmiwal, "Variable dimension filter for maneuvering target tracking," IEEE Trans. Aerospace and Electronic Systems, vol. A.ES-18, pp. 621-629, Sep 1982.

[2] N. Morrison, Introduction to Sequential Smoothing, Filtering and Prediction: McGraw Hill, 1969.

\section{APPENDIX}

\section{THEORY OF WEIGHTED AVERAGE FLTERS}

The weighted average $W_{n}$ of the function $V_{n}$ defined in equation (2) may be written as

$$
W=\frac{(1-\lambda) V_{n}}{(1-q \lambda)}
$$

where, $q$ is the backward shift operator [2].

If $x_{n}, \nabla x_{n}, \nabla^{2} x_{n}, \nabla^{3} x_{n}$ represent the current true position and the backward differences of three orders with respect to the time series of the positions, spaced at intervals $T$ apart

$$
\begin{aligned}
q^{k} x_{n}=x_{n-k}=x_{n} & -(k, 1) \nabla x_{n}+(k, 2) \nabla^{2} x_{n}-(k, 3) \nabla^{3} x_{n} \\
q^{k} \nabla x_{n} & =\nabla x_{n}-(k, 1) \nabla^{2} x_{n}+(k, 2) \nabla^{3} x_{n} \\
q^{k} \nabla^{2} x_{n} & =\nabla x_{n}-(k, 1) \nabla^{3} x_{n} \\
q^{2} \nabla^{3} x_{n} & =\nabla^{3} x_{n}
\end{aligned}
$$

where $(k, j)$ represents the binomial coefficients.

The four weighted averages of the sequence $x_{k}$ defined by the equation (8) (9) (11) and (13), corresponding to the four time constants $t_{1}$, $t_{2}, t_{3}$ and $t_{4}$ can be now evaluated. The first weighted average is

$$
A_{n}=\frac{\left(1-\lambda_{1}\right) x_{n}}{\left(1-q \lambda_{1}\right)}
$$

Expanding this as a power series in $q$ and using (A2)

$$
A_{n}=x_{n}-t_{1} \nabla x_{n}+t_{1}^{2} \nabla^{2} x_{n}-t_{1}^{3} \nabla^{3} x_{n}
$$

The first order residual is given by

$$
\left(x_{n}-A_{n}\right)=t_{1} \nabla x_{n}-t_{1}^{2} \nabla^{2} x_{n}+t_{1}^{3} \nabla^{3} x_{n}
$$

The second weighted average of these residues is given by

$$
L_{n}=\frac{\left(1-\lambda_{2}\right)\left(x_{n}-A_{n}\right)}{\left(1-q \lambda_{2}\right)}
$$

substituting for $A_{n}$ from (A7) in (A8) and expanding (A9) as a power series in $q$ and using equations (A2) to (A5), we obtain

$$
L_{n}=t_{1} \nabla x_{n}-t_{1}\left(t_{1}+t_{2}\right) \nabla^{2} x_{n}+t_{1}\left(t_{1}^{2}+t_{1} t_{2}+t_{2}^{2}\right) \nabla^{3} x_{n}
$$

$A_{n}+L_{n}$ is the position estimate of the second order filter and therefore the residue $\left(x_{n}-A_{n n}-L_{n}\right)$ is given by

$$
x_{n}-A_{n}-L_{n}=t_{1} t_{2} \nabla^{2} x_{n}-t_{1} t_{2}\left(t_{1}+t_{2}\right) \nabla^{3} x_{n}
$$

Writing

$$
D_{n}=\frac{\left(1-\lambda_{3}\right)\left(x_{n}-A_{n}-L_{n}\right)}{\left(1-q \lambda_{3}\right)}
$$

and simplifying after substitution,

$$
D_{n}=t_{1} t_{2} \nabla^{2} x_{n}-t_{1} t_{2}\left(t_{1}+t_{2}+t_{3}\right) \nabla^{3} x_{n}
$$

The third order residual is given by

$$
x_{n}-A_{n}-L_{n}-D_{n}=t_{1} t_{2} t_{3} \nabla^{3} x_{n}
$$


and therefore,

$$
E_{n}=\frac{\left(1-\lambda_{4}\right)\left(x_{n}-A_{n}-L_{n}-D_{n}\right)}{\left(1-q \lambda_{4}\right)}
$$

which simplifies to

$$
E_{n}=t_{1} t_{2} t_{3} \nabla^{3} x_{n}
$$

Combining equations (A7), (A10), (A13) and (A16) we have

$$
\left[\begin{array}{c}
A \\
L \\
D \\
E
\end{array}\right]_{n}=\left[\begin{array}{cccc}
1 & -t_{1} & t_{1}^{2} & -t_{1}^{3} \\
0 & t_{1} & -t_{1}\left(t_{1}+t_{2}\right) & t_{1}\left(t_{1}^{2}+t_{1} t_{2}+t_{2}^{2}\right. \\
0 & 0 & t_{1} t_{2} & -t_{1} t_{2}\left(t_{1}+t_{2}+t_{3}\right) \\
0 & 0 & 0 & t_{1} t_{2} t_{3}
\end{array}\right]\left[\begin{array}{c}
x \\
\nabla x \\
\nabla^{2} x \\
\nabla^{3} x
\end{array}\right]
$$

Therefore,

$$
\left[\begin{array}{c}
x \\
\nabla x \\
\nabla^{2} x \\
\nabla^{3} x
\end{array}\right]_{n}=\left[\begin{array}{cccc}
1 & 1 & 1 & 1 \\
0 & \frac{1}{t_{1}} & \frac{1}{t_{1}}+\frac{1}{t_{2}} & \frac{1}{t_{1}}+\frac{1}{t_{2}}+\frac{1}{t_{3}} \\
0 & 0 & \frac{1}{t_{1} t_{2}} & \frac{1}{t_{1} t_{2}}+\frac{1}{t_{2} t_{3}}+\frac{1}{t_{3} t_{1}} \\
0 & 0 & 0 & \frac{1}{t_{1} t_{2} t_{3}}
\end{array}\right]\left[\begin{array}{c}
A \\
L \\
D \\
E
\end{array}\right]_{n}
$$

Thus the true state vector components are obtainable from the weighted average derived from the true positions. If however we have only the measured positions available, the corresponding weighted averages are the estimates of the true weighted averages given by $[\hat{A} \hat{L} \hat{D} \hat{E}]_{n}^{T}$. Correspondingly, multiplication by the square matrix of (A18) we obtain the estimates of the state parameters $\left[\hat{x} \hat{\nabla} x \nabla^{\hat{2}} x \nabla^{\hat{3}} x\right]_{n}^{T}$, as given in equation (14).

This theory derived in general for the fourth order filter can be shortened into those for the third order or the second order filters by assuming the target dynamics defined by $\mathrm{A} 2$ to correspond to only three terms or two terms respectively, to obtain the corresponding equations (12) and (10).

Equivalence of the fourth order filter

to an $\alpha^{\prime}, \beta^{\prime}, \gamma^{\prime}, \delta^{\prime}$ filter

The $\alpha^{\prime}, \beta^{\prime}, \gamma^{\prime}, \delta^{\prime}$ filter in terms of the state vector defined in terms of difference parameters may be written as follows.

The one step predicted parameters based on state vector $\left[x_{n-1}, \nabla x_{n-1}, \nabla^{2} x_{n-1} \nabla^{3} x_{n-1}\right]^{T}$ are given by

$$
\begin{aligned}
\nabla^{3} x_{p n} & =\hat{\nabla}^{3} x_{p(n-1)} \\
\nabla^{2} x_{p n} & =\hat{\nabla}^{3} x_{n-1}+\hat{\nabla}^{3} x_{p n} \\
\nabla x_{p n} & =\hat{\nabla} x_{n-1}+\hat{\nabla}^{2} x_{p n} \\
x_{p n} & =\hat{x}_{n-1}+\nabla x_{p m}
\end{aligned}
$$

$\left(x_{M n}-x_{p n}\right)$ is the innovation for obtaining the smoothed estimate as follows.

$$
\begin{aligned}
\hat{x}_{n} & =x_{p n}+\alpha^{\prime}\left(x_{M n}-x_{p n}\right) \\
\hat{\nabla} x_{n} & =\nabla x_{p n}+\beta^{\prime}\left(x_{M n}-x_{p n}\right) \\
\hat{\nabla}^{2} x_{n} & =\nabla^{2} x_{p n}+\gamma^{\prime}\left(x_{M n}-x_{p m}\right) \\
\hat{\nabla}^{3} x_{n} & =\nabla^{3} x_{p m}+\delta^{\prime}\left(x_{M n}-x_{p n}\right)
\end{aligned}
$$

$\alpha^{\prime}, \beta^{\prime}, \gamma^{\prime}, \delta^{\prime}$ are thus the fractions of $x_{M n}$ contributed to the estimates of $x_{n}, \hat{\nabla}_{n}, \hat{\nabla}_{n}^{2}, \hat{\nabla}_{n}^{3}$ respectively at each update. Now

$$
\hat{A_{n}}=\frac{\left(1-\lambda_{1}\right)}{\left(1-q \lambda_{1}\right)} x_{M n}
$$

Therefore the contribution of the latest measurement $x_{M n}$ to $\hat{A_{n}}$ is given by $\left(1-\lambda_{1}\right)$

$$
\hat{x}_{n}-\hat{A}_{n}=\left(1-\frac{\left(1-\lambda_{1}\right)}{\left(1-q \lambda_{1}\right)}\right) x_{M n}=\frac{\lambda_{1}(1-q)}{\left(1-q \lambda_{1}\right)} x_{M n}
$$

Therefore,

$$
\hat{L}_{n}=\frac{\left(1-\lambda_{2}\right)}{\left(1-q \lambda_{1}\right)} \frac{\lambda_{1}(1-q)}{\left(1-q \lambda_{1}\right)} x_{M n}
$$

and the contribution of $x_{M n}$ to $\hat{L}_{n}$ is $\lambda_{1}\left(1-\lambda_{2}\right)$. Similarly,

$$
x_{n}-\hat{A_{n}}-\hat{L_{n}}=\frac{\lambda_{1} \lambda_{2}(1-q)^{2}}{\left(1-q \lambda_{2}\right)\left(1-q \lambda_{1}\right)} x_{M n}
$$

and therefore,

$$
\hat{D}_{n}=\frac{\left(1-\lambda_{3}\right) \lambda_{1} \lambda_{2}(1-q)^{2}}{\left(1-q \lambda_{3}\right)\left(1-q \lambda_{2}\right)\left(1-q \lambda_{1}\right)} x_{M n}
$$

The contribution of $x_{M n}$ to $\hat{D_{M n}}$ is $\lambda_{1} \lambda_{2}\left(1-\lambda_{3}\right)$. Similarly we obtain that the contribution of $x_{M n}$ to $\hat{E}_{n}$ is $\lambda_{1} \lambda_{2} \lambda_{3}\left(1-\lambda_{4}\right)$. Therefore, the contribution of $x_{M n}$ in $\hat{x}, \hat{\nabla} x, \hat{\nabla}^{2} x, \hat{\nabla}^{3} x$, may be obtained using (A18), to get $\alpha^{\prime}, \beta^{\prime}, \gamma^{\prime}, \delta^{\prime}$ values as

$$
\left[\begin{array}{c}
\alpha^{\prime} \\
\beta^{\prime} \\
\gamma^{\prime} \\
\delta^{\prime}
\end{array}\right]=\left[\begin{array}{cccc}
1 & 1 & 1 & 1 \\
0 & \frac{1}{t_{1}} & \frac{1}{t_{1}}+\frac{1}{t_{2}} & \frac{1}{t_{1}}+\frac{1}{t_{2}}+\frac{1}{t_{3}} \\
0 & 0 & \frac{1}{t_{1} t_{2}} & \frac{1}{t_{1} t_{2}}+\frac{1}{t_{2} t_{3}}+\frac{1}{t_{3} t_{1}} \\
0 & 0 & 0 & \frac{1}{t_{1} t_{2} t_{3}}
\end{array}\right]\left[\begin{array}{c}
1-\lambda_{1} \\
\lambda_{1}\left(1-\lambda_{2}\right) \\
\lambda_{1} \lambda_{2}\left(1-\lambda_{3}\right) \\
\lambda_{1} \lambda_{2} \lambda_{3}\left(1-\lambda_{4}\right)
\end{array}\right]
$$

The equation provides the equivalence of the fourth order filter to an $\alpha^{\prime}, \beta^{\prime}, \gamma^{\prime}, \delta^{\prime}$ filter.

Equivalence of the third order filter and the second order filter to the corresponding $\alpha^{\prime}, \beta^{\prime}, \gamma^{\prime}$ filter or $\alpha^{\prime}, \beta^{\prime}$ filter may be similarly obtained by truncating the above equation to $3^{\text {rd }}$ or $2^{\text {nd }}$ order matrices respectively.

It may be seen that when $\lambda_{1}=\lambda_{2}=\lambda_{3}=\lambda_{4}$, the filters degenerate into the least square fading memory filters of the corresponding orders.

\section{Variance Reduction Factor $K_{D}$ of $D_{n}$}

Expanding equation (A.31) as a power series in $q$ and associating an error $\sigma_{x}^{2}$ with each measured value $x_{M(n-k)}$ (for $\mathrm{k}=0$ to $\infty$ ), we obtain the variance reduction ratio $K_{D}$ for $D_{n}$ as given in equation (18)

Variance Reduction Factor $K_{E}$ of $E_{n}$

In a similar manner, writing

$$
E_{n}=\frac{\left(1-\lambda_{4}\right) \lambda_{1} \lambda_{2} \lambda_{3}(1-q)^{3}}{\left(1-q \lambda_{4}\right)\left(1-q \lambda_{3}\right)\left(1-q \lambda_{2}\right)\left(1-q \lambda_{1}\right)} x_{M_{n}}
$$

we obtain an expression for $K_{E}$. The algebraic operations are too voluminous and cannot be easily attempted without a computer aid. Only the final result is given below.

$$
\begin{aligned}
& K_{E}=\frac{\sigma_{E}^{2}}{\sigma_{x M}^{2}} \\
& =\frac{\left(1-\lambda_{4}^{2}\right) \lambda_{1}^{2} \lambda_{2}^{2} \lambda_{3}^{2} f\left(\lambda_{1} \lambda_{2} \lambda_{3} \lambda_{4}\right)}{\left(1-\lambda_{1} \lambda_{2}\right)\left(1-\lambda_{1} \lambda_{3}\right)\left(1-\lambda_{1} \lambda_{4}\right)\left(1-\lambda_{2} \lambda_{3}\right)\left(1-\lambda_{2} \lambda_{4}\right)\left(1-\lambda_{3} \lambda_{4}\right) \Pi\left(1+\lambda_{1}\right)}
\end{aligned}
$$

where,

$$
\begin{aligned}
f\left(\lambda_{1}, \lambda_{2}, \lambda_{3}, \lambda_{4}\right)= & 2 \lambda_{1} \lambda_{2} \lambda_{3} \lambda_{4}\left\{12 \sum\left(\frac{1}{\lambda_{1}}-\lambda_{1}\right)\right. \\
& +\sum\left(\frac{\lambda_{1}}{\lambda_{2} \lambda_{3}+\lambda_{3} \lambda_{4}+\lambda_{4} \lambda_{2}}-\frac{\lambda_{2} \lambda_{3}+\lambda_{3} \lambda_{4}+\lambda_{4} \lambda_{2}}{\lambda_{1}}\right) \\
& +\sum\left(\frac{\lambda_{1}}{\lambda_{2} \lambda_{3} \lambda_{4}}-\frac{\lambda_{2} \lambda_{3} \lambda_{4}}{\lambda_{1}}\right) \\
& -2 \sum\left(\frac{1}{\lambda_{1} \lambda_{2}}+\frac{1}{\lambda_{1} \lambda_{3}}+\frac{1}{\lambda_{1} \lambda_{4}}-\lambda_{1}\left(\lambda_{2}+\lambda_{3}+\lambda_{4}\right)\right) \\
- & \left.5 \sum\left(\frac{1}{\lambda_{1} \lambda_{2} \lambda_{3}}-\lambda_{1} \lambda_{2} \lambda_{3}\right)+10\left(\frac{1}{\lambda_{1} \lambda_{2} \lambda_{3} \lambda_{4}}-\lambda_{1} \lambda_{2} \lambda_{3} \lambda_{4}\right)\right\}
\end{aligned}
$$

The summation $\sum$ and the product $\Pi$ in the above are cyclic over the indices $1,2,3$ and 4 .

The values $K_{D}$ and $K_{E}$, as required in the algorithm, are precomputed using the above expressions. 